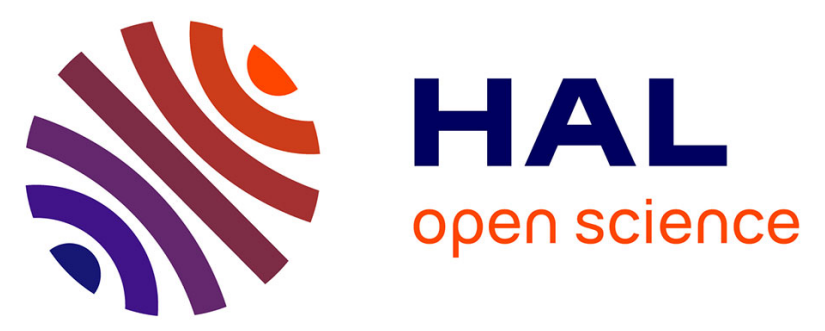

\title{
The LoCoSS project of GIS ITS Bretagne: status and perspectives
}

Fabrice Comblet, François Peyret, Cyril Ray, Jean-Marie Bonnin, Yvon Le Roux

\section{- To cite this version:}

Fabrice Comblet, François Peyret, Cyril Ray, Jean-Marie Bonnin, Yvon Le Roux. The LoCoSS project of GIS ITS Bretagne: status and perspectives. 14th World Congress on Intelligent Transport Systems, Oct 2007, Beijing (Pékin), China. hal-02900954

\section{HAL Id: hal-02900954 https://hal.science/hal-02900954}

Submitted on 16 Jul 2020

HAL is a multi-disciplinary open access archive for the deposit and dissemination of scientific research documents, whether they are published or not. The documents may come from teaching and research institutions in France or abroad, or from public or private research centers.
L'archive ouverte pluridisciplinaire HAL, est destinée au dépôt et à la diffusion de documents scientifiques de niveau recherche, publiés ou non, émanant des établissements d'enseignement et de recherche français ou étrangers, des laboratoires publics ou privés. 


\title{
The LoCoSS project of GIS ITS Bretagne: status and perspectives
}

\author{
Fabrice Comblet $^{* * *}$, François Peyret ${ }^{*}$, Cyril Ray ${ }^{* *}$, Jean-Marie Bonnin ${ }^{* * * *}$, Yvon-Marie Le Roux ${ }^{* * * * *}$ \\ * Laboratoire Central des Ponts et Chaussées, BP 4129, 44341 BOUGUENAIS Cedex, France \\ francois.peyret@lcpc.fr \\ ** French Naval Academy Research Institute, 29240 BREST Armées, BP600, France \\ ray@ecole-navale.fr \\ ****Ecole Nationale des Ingénieurs des Etudes et Techniques d’Armement, 2 rue François Verny \\ 29806 BREST Cedex 9, France \\ comblefa@ensieta.fr \\ * GET-ENST Bretagne, Technopole Brest Iroise, CS 83818, 29238 BREST, France \\ jm.bonnin@enst-bretagne.fr, yvon.leroux@enst-bretagne.fr
}

\begin{abstract}
A scientific coordination group devoted to Intelligent Transportation Systems has been set up in the Brittany region, called GIS ITS Bretagne. The focus of this paper is on the LoCoSS project, one of the two first projects launched by the GIS, which aims at improving the efficiency and safety of terrestrial and maritime rescue operations through the use of geolocalization and wireless communication technologies. The paper gives an overview of the project and presents its progress after the first year, particularly in terms of geographical data management and indoor localization of the intervening firemen.
\end{abstract}

\section{INTRODUCTION OF THE GIS ITS-BRETAGNE}

The existence of a significant research task force in computer science, computer engineering, electronics, signal processing and telecommunications in Brittany has recently led to the setting of the scientific interest group called GIS ITS Bretagne devoted to Intelligent Transportation Systems. It gathers research units of universities (Rennes and Nantes), graduate engineering schools (ENST Bretagne, ENSSAT, ENSIETA, INSA de Rennes), the Ministry of Transports (CETE, LCPC) and France Telecom R\&D. The GIS was officially launched in February 2005.

Its main objective is to stimulate and federate research projects as well as innovative actions and experiments using technological platforms. Moreover, it looks for synergies with other structures at the regional, national or international levels. It is strongly related to social needs in particular security issues by means of technologies or services (e.g., using embedded devices). One of its important missions is to attract and associate (big, medium range and small) companies in the R\&D activities which will be undertaken.

The ITS domain is broad and the first discussions have focused on the identification of a priority: services to mobile users. This theme covers several issues and the two first projects of the GIS that have been recently initiated, have received funding by the Regional Council of Brittany. One of those two is called CAPTIV and aims at studying and developing intelligent signaling systems capable to transmit and display the road signs on-board of the vehicles. The LoCoSS project, that which will be more precisely detailed in the paper, deals with geolocalization, geographical information systems and wireless communications applied to disaster response management and coordination.

\section{OVERVIEW OF THE LOCOSS PROJECT}

LoCoSS aims at bringing significant improvements to the rescue brigades operations, terrestrial as well as maritime, thanks to ITS technologies.

In the case of an accident or a disaster occurring either on the road, in a factory, in a city, in a harbor or on a boat cruising close to the coast, three points are of the highest importance: the rapidity of the intervention of the brigade, the exactness, the completeness and up-to-date character of the critical information which is available at the level of the headquarters vehicle and the possibility for the intervening agents to remain in permanent contact with it. The idea of the project is to combine the three basic ITS technologies, i.e. wireless communications, geopositioning technologies and geographic information systems (GIS), to facilitate and improve the above mentioned points. 
Numerous wireless communication technologies standards (GPRS, Wi-Fi, WiMAX, DSRC, UMTS...) could be optimally used in order to offer a permanent IPv6 connectivity through NEMO technology (4)(5). Therefore, the commanding vehicle will be able to download continuously before the departure to the site, during its travel and during the operation, the necessary information into its local database to steer efficiently the operations. Wireless bidirectional links will also be established between the vehicle and some members of the team who could be equipped with a positioning pack, in order to trace their movements from the vehicle and to optimize the operations management. Since all pieces of equipment (in the vehicle and on the member of the team) will be provided with a full IPv6 connectivity, the commanding vehicle will be allowed to directly request information from them.

A specific piece of hardware (the mobile router) will be devoted to network connectivity management in the vehicle. It will be in charge of managing different network interfaces (7) in order to provide firemen and embarked applications with the best feasible connectivity to the Internet (Figure 1). Several interfaces could be used simultaneously to improve the available bandwidth and reduce the interruption time when one network becomes unreachable (6). This mobile router will also use common IP priority mechanisms to serve critical applications first (8).

As some rescue operation could stand in an area without any network connectivity, the nested NEMO (4) will be used to "relay" the traffic from the out of range area to the Internet (Figure 1). One vehicle will remain in the range of whichever network and let the vehicle in operation attach to it. This way all vehicles operate as usual, using NEMO to provide homelike operation to all nodes attached to it.
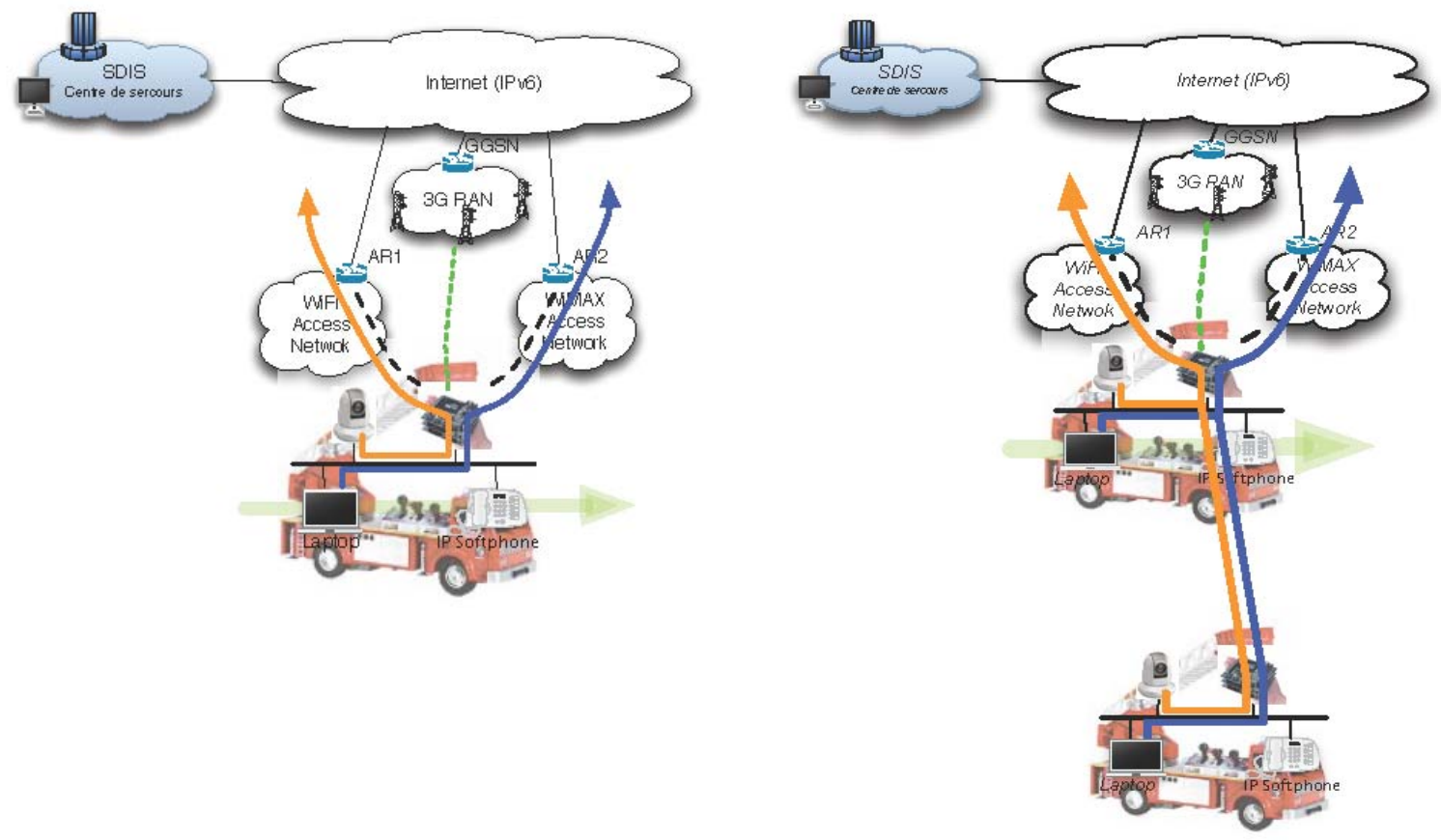

Figure 1. Mobile router managing network connectivity (left), nested case (right)

Thanks to the positioning technologies, based on EGNOS, aided by additional sensors to ensure a high level of integrity, the intervening vehicles will be able to navigate precisely and accurately up to the accident or disaster location and the main stationary headquarters (typically the fire station) will be informed precisely about their progress. For the difficult issue of tracking the firemen inside a big site such as a factory of a ship, novel positioning techniques such as high-sensitivity or assisted GPS receivers, combined with inertial sensors and 3D map-matching will be developed and experimented.

Finally, on top of the geolocalisation and communication infrastructure, a Geographical Information System (GIS) will act as computing software able to integrate, store, model, 
analyze and visualize spatially related data (2) (3). The information system of the LoCoSS project will be used to build and to update an on-board database limited to the necessary information to support the operations in a given area. This "mobile database" will have to be extracted from the main one at security headquarters. Similarly, the main database located at the fire station will have to be interactively updated in real-time during the progress of the rescue operations. Amongst the interesting dynamic data to integrate within databases are firemen and vehicles positions and movements. The continuous recording of such data is essential for real-time following of actual operations.

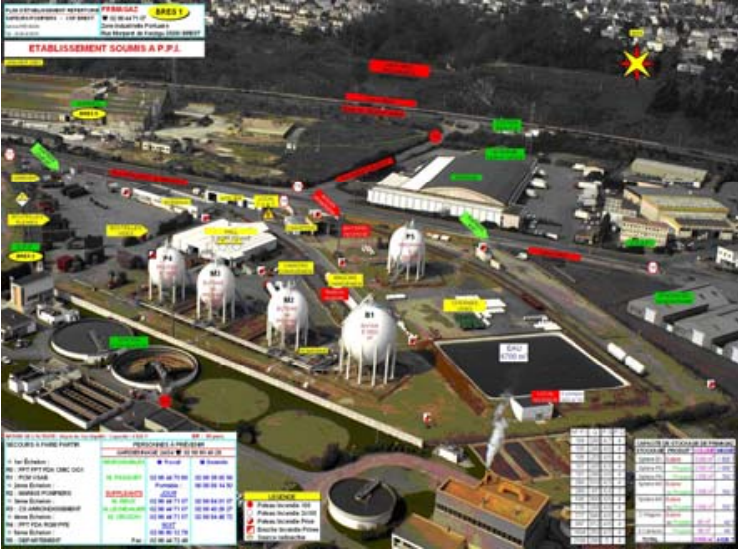

(a)

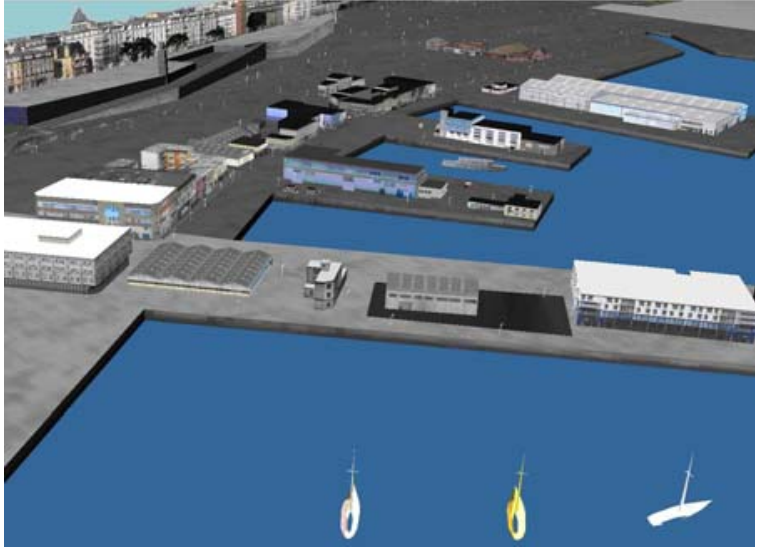

(b)

Figure 2. 3D simulation environment of an industrial area (a) and Brest harbour (b)

\section{III.PROGRESS OF THE LOCOSS PROJECT}

At the end of the first year of the project, the partners have mainly worked on the following items: functional and technical specifications and feasibility pre-study on the positioning aspects. For establishing the specifications, frequent meetings were organized between the project partners and the fire brigades of Côtes d'Armor and Finistère departments. Several use cases were chosen, particularly relevant for the firemen.

In terms of information systems, the firemen already have at their headquarters a software platform (cf. figure 2) that is still under development but that already offers a high level of functionality in terms of data integration and visualization. The platform includes around 20 sensitive areas with all the relevant information in terms of map data, aerial photographs, access plans, etc. as well as administrative, juridical and methodological information. The platform also includes 3D virtual reality models of some of these areas. But this platform remains static in the sense it does not allow a real-time monitoring of operations. The objective of the LoCoSS project is to add and manage this dynamic (and real-time) dimension within firemen's platform.

For that purpose, the project will develop mechanisms to acquire the real-time data coming from the geo-localized vehicles and people through the wireless communication links and to store them in an appropriate database. Spatio-temporal queries to database will be developed to handle and display these dynamic data on the existing firemen's interface. Overall objective will be to visualize the progress of the deployed forces and to make decisions in real-time thanks to the global view of the situation. The LoCoSS information system will be also extremely profitable after the operation, where GIS can be used to replay operations, for training and learning purposes.

As far as research on positioning is concerned, partner ENSIETA is presently investigating different positioning technologies that could be utilized for the challenging indoor navigation. The basic idea is to combine several technologies: high-sensitivity GNSS receivers for the areas where GNSS signals are penetrating, MEMS inertial measurement units for the traveled distance based upon the number and size of steps, the direction provided by the gyros and magnetometers and advanced map-matching algorithms based upon the 2D 
or 3D map of the area and the topological constraints (1). First results from the LoCoSS project concern the firemen localization in constrained environment (forest, indoor, city...) where GPS position is not precise enough. We have been interested in different positioning technologies able to increase the GPS localisation in these environments. The solution we adopted uses MEMS inertial measurements units for relative localization. The travelled distance is obtained from a vertical accelerometer for the number and size of steps, the direction is provided by gyros and magnetometers. Figure 3 illustrates the real trajectory done inside one of the ENSIETA building and figure 4 presents the obtained results (raw data from MEMS inertial sensors).

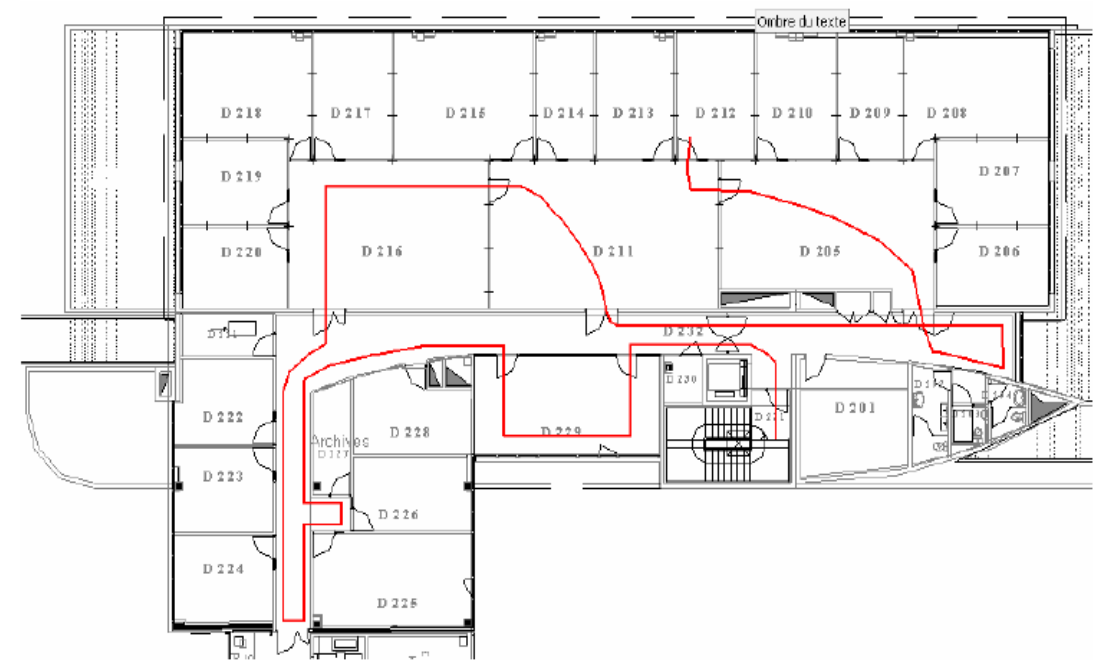

Figure 3. Real trajectory while using a MEMS IMU

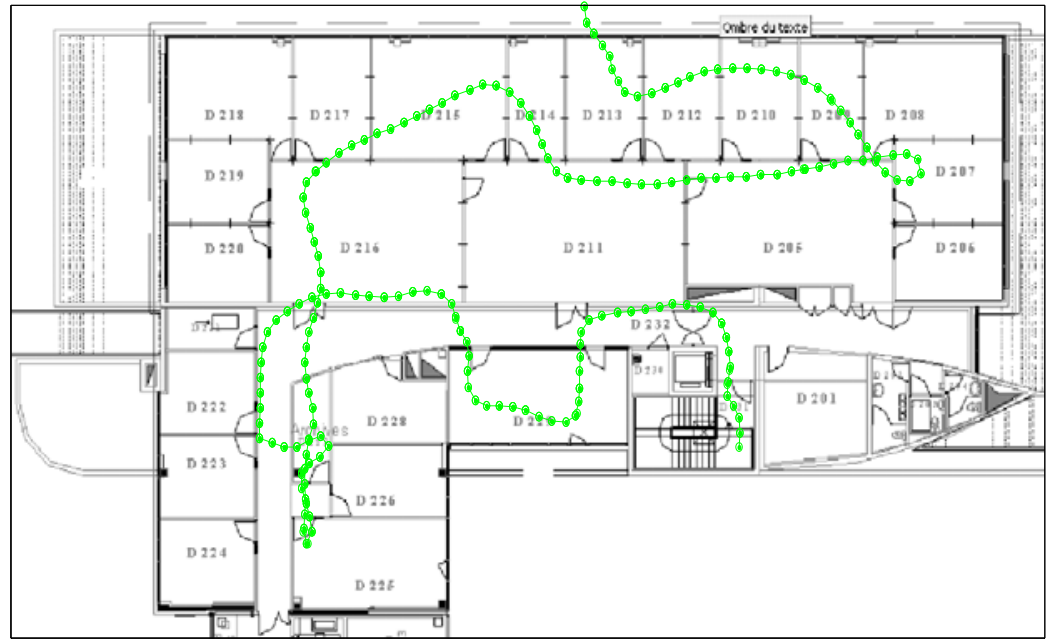

Figure 4. Estimated trajectory by the MEMS IMU

In figure 4, the approximate way can be found from the estimated trajectory, but the final position obtained is far from the real one. This position error is due to MEMS measurements errors which lead to a trajectory deviation.

In outdoor environments, a valid GPS position is sometimes possible. So we can use this position to readjust position given from MEMS measurements and reduce the final error. In indoor environment, the navigation is more complex because GPS is not operational and 
can not be used to readjust position. In order to readjust indoor position we develop an indoor map-matching algorithm based on Monte Carlo method. First, we need a precise building referencing with all accessible positions by firemen (see figure 5.a) in order to know precisely, at a given time, the rule constraining accessible positions from current estimated location. The selected grid is an 8-connexcity with $0.5 \mathrm{~m}$ step which permits to describe all types of building. This grid allows having precise information on firemen positions to rescue them in the event of problem. Then, from these different accessible positions we define all available directions which determinate the evolution law of our algorithm. Figure 5.b illustrates available direction (green arrow) in free space and in a corner. The proscribed directions are in red.

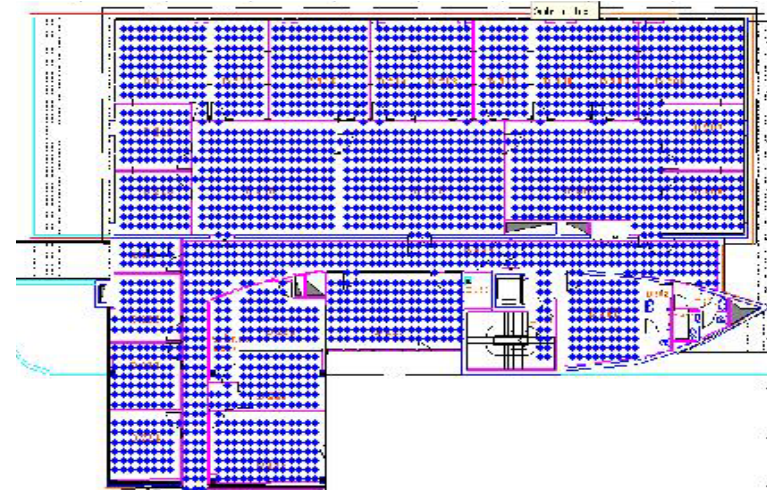

(a) : used grid

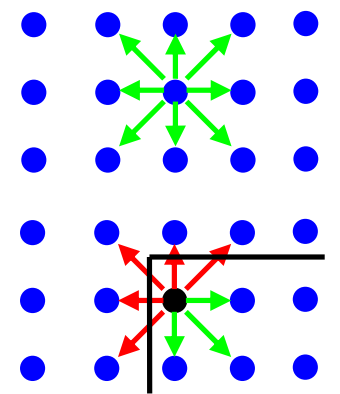

(b) : evolution law in free space and in a corner

Figure 5. Building referencing

Finally, the developed algorithm evolution is based on Monte Carlo method. We defined a valid whole of position from the start position. The positions evolution is obtained from MEMS measurements, MEMS error and the evolution law. This algorithm allows obtaining the real trajectory realised by the firemen and giving a good estimation of the final position (figure 6).

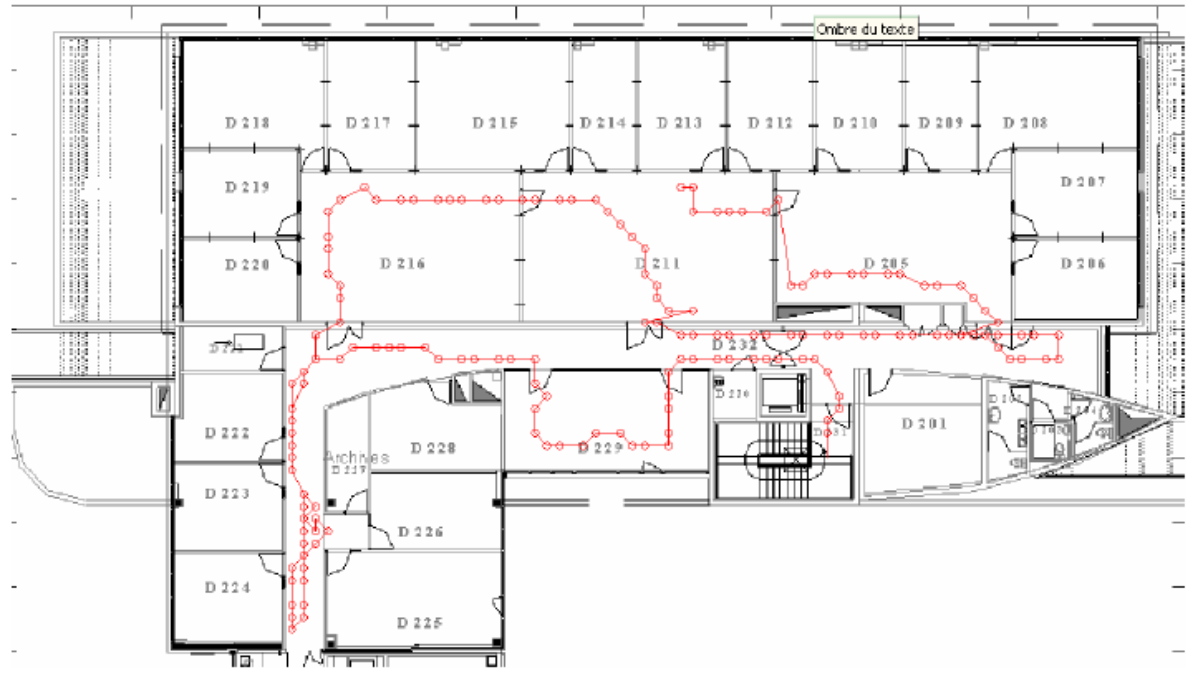

Figure 6. Trajectory estimation by a MEMS IMU and dedicated map-matching algorithm

\section{ACKNOWLEDGMENT}

The LoCoSS project is partially funded by the Brittany Council and by the Laboratoire Central des Ponts et Chaussées. 


\section{REFERENCES}

(1) Gillieron P.Y., Spassov I. and Merminod B., "Indoor navigation enhanced by mapmatching", European Journal of navigation, 3(3), 2005

(2) Goodchild, M. F., "GIS and Transportation: Status and Challenges", GeoInformatica, 4, pp. 127-139, 2000.

(3) Thill, J. C., "GIS in Transportation Research", Pergamon Press, Oxford, UK, 2001

(4) V. Devarapalli, R. Wakikawa, A. Petrescu, and P. Thubert. NEtwork Mobility (NEMO) Basic Support Protocol, RFC 3963, IETF. January 2005.

(5) R. Wakikawa, K. Uehara, T. Ernst, and K. Nagamix. Multiple Care-of Address Registration, IETF draft-wakikawa-mobileip-multiplecoa-05.txt (work in progress). February 2006.

(6) T. Ernst and J. Charbon. Multihoming with Nemo Basic Support. In First International Conference on Mobile Computing and Ubiquitous (ICMU), Yokosuka, Japan, January 2004.

(7) L. Suciu, J.-M. Bonnin, K. Guillouard, and T. Ernst. Multiple Network Interfaces Management for Mobile Routers. In 5th International Conference on ITS Telecommunications (ITST 2005), Brest, France, June 2005.

(8) M. Mahamat-faki, J.-M. Bonnin, and R. Ben Rayana. Traffic flow control on multitunneled mobile router. In short paper in WNEPT06 (Workshop on Networking in Public Transport), Waterloo, Canada, August 2006. 